\title{
Association between dietary fibre intake and asthma (symptoms and control): results from the French national e-cohort NutriNet-Santé
}

\author{
Roland M. Andrianasolo ${ }^{1 *}$, Serge Hercberg ${ }^{1,2}$, Emmanuelle Kesse-Guyot $^{1}$, Nathalie Druesne-Pecollo ${ }^{1}$, \\ Mathilde Touvier ${ }^{1}$, Pilar Galan ${ }^{1}$ and Raphaëlle Varraso ${ }^{3,4}$ \\ ${ }^{1}$ Université Paris 13, Equipe de Recherche en Epidémiologie Nutritionnelle (EREN), Centre de Recherche en Epidémiologie \\ et Statistiques - Université de Paris, Inserm (U1153), Inra (U1125), Cnam, Bobigny, France \\ ${ }^{2}$ Département de Santé Publique, Hôpital Avicenne, Bobigny, France \\ ${ }^{3}$ Inserm, U1168, VIMA: Aging and chronic diseases. Epidemiological and public health approaches, Villejuif F-94807, France \\ ${ }^{4}$ Université Versailles St-Quentin-en-Yvelines, UMR-S 1168, Montigny le Bretonneux F-78180, France \\ (Submitted 15 March 2019 - Final revision received 12 July 2019 - Accepted 18 July 2019)
}

\section{Abstract}

Despite growing evidence suggesting a potential health benefit of high fibre intake for the prevention of chronic inflammatory diseases, studies regarding the role of total dietary fibre intake, types and sources of fibre on asthma are lacking. The present study aimed to investigate the crosssectional association of dietary fibre intakes and source of fibre with the asthma symptom score and asthma control. A total of 35380 participants from the NutriNet-Santé cohort were included. Asthma was defined by the asthma symptom score and asthma control by the asthma control test. Fibre intake $(\mathrm{g} / \mathrm{d})$ was categorised according to sex-specific quintiles. Multi-adjusted negative binomial regressions were used to evaluate the association between dietary fibre with the asthma symptom score and logistic regressions with asthma control. Participants were aged on average 54 years. After adjustment for confounders, higher intake of total, soluble, insoluble fibres from cereals, fruit and seeds were significantly negatively associated with the asthma symptom score both among women and men; OR for the highest quintile of total dietary fibre compared with the lowest quintile were 0.73 (95\% CI $0.67,0.79)$ in women and $0.63(95 \% \mathrm{CI} 0.55,0.73)$ in men. We also found inverse significant associations between total, soluble and insoluble fibre with uncontrolled asthma; OR for participants in the highest quintile of total dietary fibre was $0.72(95 \%$ CI $0.55,0.95)$ in women and $0.45(95 \%$ CI $0.26,0.79)$ in men. Our results suggested that higher intake of dietary fibre, mostly insoluble fibre and fibre from cereals, was associated with fewer asthma symptoms and greater asthma control.

Key words: Dietary fibre: Soluble fibre: Insoluble fibre: Cereal fibre: mPNNS-GS: Asthma symptom score: Asthma control

Dietary guidelines across the world have provided consistent recommendations regarding the increased intake of fruits, vegetables or whole grains ${ }^{(1-3)}$. Although several components of the diet may be involved in the potential health benefit, fruit, vegetables, legumes and whole grains are thought to play a role through their high concentrations of antioxidant vitamins and fibre $^{(4)}$. Epidemiological studies have widely reported that a diet rich in fruit, vegetables and whole grains is associated with a reduced risk of several chronic diseases ${ }^{(4-9)}$. More specifically, regarding dietary fibre intake, many studies have reported the importance of its consumption for health, to prevent chronic diseases such as $\mathrm{CVD}^{(10)}$, type 2 diabetes $^{(11)}$ and some cancers ${ }^{(12)}$.

Regarding lung health, dietary fibre intake has been associated with a lower risk of respiratory disease mortality ${ }^{(13)}$, a greater lung function ${ }^{(13,14)}$ and a decreased risk of chronic obstructive pulmonary disease (COPD) $)^{(15,16)}$ and COPD symptoms ${ }^{(17)}$. Diets low in dietary fibre have been shown to affect the immune system and are thought to promote the development of a variety of immune disorders, including allergies and asthma $^{(18)}$. To our knowledge, two studies have been conducted to evaluate the association between dietary fibre and asthma in adults $^{(19,20)}$. Berthon et al. reported an inverse association between total fibre intake and severe persistent asthma ${ }^{(19)}$, and Halnes et al. an inverse association between soluble fibre intake and airway inflammation in asthma ${ }^{(20)}$. Both studies were performed among asthmatics only and without investigating different types nor sources of fibre.

Asthma is a heterogeneous disease with different phenotypes, variable clinical manifestations and temporal phenotypic variability $^{(21)}$. Thus, rather than dichotomous definition of

Abbreviations: ACT, asthma control test; COPD, chronic obstructive pulmonary disease; mPNNS-GS, modified Programme National Nutrition Santé-guidelines score.

* Corresponding author: R. M. Andrianasolo, email r.andrianasolo@eren.smbh.univ-paris13.fr 
asthma, such as doctor-diagnosed asthma, in epidemiological studies, it is necessary to be able to capture the complexity of asthma. The asthma symptom score has been recommended for the study of risk factors in asthma ${ }^{(22,23)}$ and is particularly relevant when investigating association with diet, as it can be used for both participants with and without asthma allowing integration of different asthma phenotypes and different levels of asthma prevention. Likewise, achieving and maintaining a good asthma control is the main goal of asthma management, and better understanding as to why asthma is getting worse (or better) in a substantial proportion of patients would be very informative. To our knowledge, no previous studies have been conducted to investigate the potential role of different types and sources of dietary fibre both on asthma symptoms and on asthma control.

The objectives of the present study conducted in a large cohort of French adults were to (1) investigate the cross-sectional association of total dietary fibre, soluble and insoluble fibre and different sources of fibre (cereals, vegetables, fruit, seeds and legumes) with the asthma symptom score and (2) further evaluate the impact of dietary fibre consumption on asthma control.

\section{Methods}

\section{Population}

Subjects were participants of the NutriNet-Santé Study, an ongoing web-based prospective observational French cohort of volunteers aged 18 years and older, launched in May 2009. Briefly, its main objective is to investigate the relationship between nutrition and health as well as of determinants of dietary behaviours and nutritional status. At enrolment, participants complete a baseline set of questionnaires; and during the follow-up, they receive monthly automated emails informing that new questionnaires are available in the secured platform. More detailed information has been published elsewhere ${ }^{(24)}$. The NutriNet-Santé study is conducted according to the Declaration of Helsinki guidelines and was approved by the Institutional Review Board of the French Institute for Health and Medical Research (IRB Inserm no. 0000388FWA00005831) and the 'Commission Nationale de l'Informatique et des Libertés' (CNIL no. 908450/no. 909216). It is registered at clinicaltrials.gov as NCT03335644. All participants provided electronic informed consent.

\section{Dietary data collection}

Participants were invited to complete three non-consecutive self-administered web-based 24-h dietary records randomly distributed within a 2-week period at baseline and every 6 months. Self-administered web-based 24-h dietary records have been validated against urinary ${ }^{(25)}$ and plasma biomarkers ${ }^{(26)}$ and against interview by a trained dietitian ${ }^{(27)}$.

Participants reported all foods and beverages at each eating occasions during the day. Portion size for each foods and beverages were directly entered as grams or volumes or estimated using previously validated photographs ${ }^{(28)}$. Daily dietary intake was calculated as the average of at least three 24-h dietary records. Energy underreporting participants were identified and excluded using the validated method developed by Black ${ }^{(29)}$. Black's equations are based on an estimate of the person's BMR calculated via Schofield's equations ${ }^{(30)}$ and taking into account sex, age, height and weight as well as the physical activity level (PAL), number of 24-h records, intra-individual variabilities of reported energy intake and BMR and intra-/ inter-variabilities of PAL.

Dietary fibre intake was calculated in $\mathrm{g} / \mathrm{d}$, and we considered total dietary fibre, soluble and insoluble fibres (types of fibre) and cereal fibre, vegetable fibre, fruit fibre, seed fibre and legume fibre (sources of fibre). We obtained the dietary fibre content of each food from the NutriNet-Santé food composition table ${ }^{(31)}$, which is a compilation of data from the Supplementation en Vitamines et Minéraux AntioXydants (SU.VI.MAX) study food database, published literature and other food composition tables from different European countries. For mixed dishes, fibre contents were calculated using nutrient contents of individual foodstuffs and usual recipes. Dietary fibre intake (total, types and sources) was adjusted for total energy intake using the residual method ${ }^{(32)}$ and was classified based on sex-specific quintiles.

To assess the overall nutritional quality of the diet, we computed the modified Programme National Nutrition Santéguidelines score (mPNNS-GS), without physical activity, reflecting adherence to the French nutritional recommendations. The mPNNS-GS includes twelve components: eight components referred to food-serving adequacy and four referred to moderation in consumption. Moreover, points are deducted for overconsumption of salt, added sugars or when energy intake exceeds the estimated energy needs by more than $5 \%$. Scoring has been described in detail elsewhere ${ }^{(33)}$, and the final score ranges from (possible) negative scores to $13 \cdot 5$.

\section{The respiratory survey in the NutriNet-santé cohort}

To improve the respiratory characterisation of the participants of the cohort, a non-mandatory detailed questionnaire on respiratory health based on international standardised recommendations ${ }^{(34)}$ was proposed in April 2016 to 121568 active participants included in the cohort at this date. As of June 2017, the survey was filled in by 40152 adults. Respondents ( $n 40152$ ) were significantly older as compared with non-respondents ( $n 81$ 416). After adjustment for age, they were also more likely never smokers, had a higher educational level, were more physically active and less likely to be obese $^{(35)}$. Ever asthma was defined by at least one positive answer to the question 'Have you ever had asthma?' in main questionnaires or by a positive answer to 'Have you ever had an asthma attack?' or 'Have you ever had an attack of shortness of breath at rest with wheezing' in the respiratory survey.

We used the asthma symptom score ${ }^{(22,23)}$ which has been proposed previously as a continuous measure of asthma in epidemiological studies. It is a validated score, ranging from 0 to 5 , based on the number of respiratory symptoms during the past 12 months.

The asthma control test (ACT) ${ }^{(36)}$ was used to evaluate asthma control. It is a validated self-administered questionnaire based on five questions on activity limitations, frequency of symptoms, frequency of use of quick-relief medication and the subjective 
perception of the level of asthma control during the previous 4 weeks. Each item is scored from 1 to 5 , and the total ACT score ranges from 5 to 25 . Participants with asthma were classified as well-controlled (ACT $>19)$ or uncontrolled $(\text { ACT } \leq 19)^{(36)}$.

Allergic rhinitis was defined as a positive answer to 'Have you ever had allergic rhinitis? or 'Have you ever had hay fever?' Information about family history of asthma was also collected (yes/no).

\section{Assessment of covariates}

At baseline, validated self-administered questionnaires were used to collect data which includes sex, age, educational level $(<13,14,15-16, \geq 17$ years) and smoking status (never smokers, ex-smokers, current smokers). Among ever smokers, pack-years were calculated to estimate the amount of tobacco smoke. BMI was calculated as weight $(\mathrm{kg}) /$ height $^{2}$ (in $\mathrm{m}^{2}$ ) and categorised according to the WHO classification $(<18 \cdot 5$, $\left.18 \cdot 5-24, \quad 25-29, \geq 30 \mathrm{~kg} / \mathrm{m}^{2}\right)^{(37)}$. Self-reported leisure-time physical activity was assessed using the French short form of the International Physical Activity Questionnaire ${ }^{(38)}$. The weekly activity-associated energy expenditure expressed in metabolic equivalent task in minutes per week was estimated, and three categories of physical activity were defined (vigorous $(\geq 60 \mathrm{~min} / \mathrm{d}$ ), moderate $(30-59 \mathrm{~min} / \mathrm{d}$ ) and low $(<30 \mathrm{~min} / \mathrm{d}))$.

\section{Statistical analysis}

From the 40152 participants who filled in the respiratory survey, we included those with at least three dietary records from baseline till their 2 years of follow-up, that is, mean $=8 \cdot 0$ ( $\mathrm{sD} 3 \cdot 0)$, and who were not identified as energy underreporters to account for intra-individual variation in intake and estimate usual intake. Our analytic population included 26640 women and 8740 men (online Supplementary Fig. S1). Regarding asthma control, 2094 women and 557 men completed the ACT questionnaire out of the 3116 participants who reported ever asthma.

All analyses were conducted separately among women and men since the effect of dietary fibre may differ in women and men ${ }^{(39)}$. As previously done for this score, association between quintiles of total fibre, types and sources with the asthma symptom score (treated as a continuous variable) were evaluated using a binomial negative regression mod$\mathrm{els}^{(40)}$. Multivariable models were adjusted for age, smoking status, pack-years in smokers, educational level, leisure-time physical activity, total daily energy intake, allergic rhinitis and family history of asthma. Tests for linear trend were performed using median value for each quintiles of fibre intake as a continuous variable in the model.

We performed several sensitivity analyses. First, we tested whether the association between dietary fibre intake and the asthma symptom score was modified by smoking status, and we formally tested the interaction between fibre intake and smoking. Second, since growing evidence suggests that dietary fibre is associated with overweight and obesity in adults ${ }^{(41)}$, and BMI a likely potential mediator in the diet-asthma association ${ }^{(42)}$, we performed further analysis stratified by BMI and tested the interaction between fibre intake and BMI. Then since a high fibre intake might reflect an overall healthy diet, and to account for the overall remaining effect of diet in the fibreasthma association, we performed a sensitivity analysis by adjusting for overall nutritional quality of diet assessed by the mPNNS-GS, a score reflecting adherence to French nutritional recommendations ${ }^{(33)}$. We also performed analysis stratified across mPNNS-GS sex-specific tertiles. Finally, we performed a sensitivity analysis among participants who never reported asthma from their inclusion in the NutriNetSanté cohort till their answer to the respiratory survey (a likely free of asthma population).

For asthma control, association with quintiles of dietary fibre (total, types and sources) was evaluated using logistic regression. Main models were adjusted for age, smoking, pack-years, educational level, leisure-time physical activity and total daily energy intake. Further analysis was also performed by adjusting for the overall nutritional quality of diet assessed by the mPNNS-GS.

Missing data for covariates were handled by multiple imputations ( $n$ 10), according to a Markov chain Monte Carlo $\operatorname{approach}^{(43)}$.

We used SAS software version 9.4 (SAS Institute) to analyse the data. All the tests were two sided and the significance level was set at $0 \cdot 05$.

\section{Results}

The descriptive characteristics of the participants according to quintiles of energy-adjusted total dietary fibre are shown in Table 1 . The mean age was $53^{(14)}$ years in women and $59^{(13)}$ years in men. The average dietary fibre intake was $20.3 \mathrm{~g} / \mathrm{d}$ among women and $20.5 \mathrm{~g} / \mathrm{d}$ among men. Main contributors to total fibre intake were cereals ( $29 \%$ in women and $31 \%$ in men), vegetables (26\% in women and $25 \%$ in men), fruits (20\% both in women and men), legumes ( $3 \%$ in women and $4 \%$ in men) and nuts ( $2 \%$ both in women and men). Total dietary fibre, soluble and insoluble fibre intakes were highly correlated with dietary fibre intake from fruit and legumes both among women (online Supplementary Table S1) and men (online Supplementary Table S2). Among women and men, participants in the highest quintile of total dietary fibre were significantly older, less likely to be current smokers, more physically active during leisure time, had a higher educational level and were less likely to be obese than participants in the lowest quintile of total dietary fibre.

\section{Types of dietary fibre and asthma symptom score}

In our study, $28 \%$ of women and $25 \%$ of men reported at least one asthma symptom. After adjustment for potential confounders, total dietary fibre intake was significantly negatively associated with asthma symptoms among both women and men (Fig. 1). The OR for the participants in the highest quintile of total dietary fibre was $0.73(95 \%$ CI $0.67,0.79)$ in women (Table 2) and 0.63 (95\% CI 0.55, 0.73) in men (Table 3) compared with participants in the lowest quintile of total dietary fibre intake, with a significant trend $(P<0 \cdot 0001)$. Further adjustment for the mPNNS-GS did not modify the association (online Supplementary Tables S3 and S4).

After stratification on smoking habits, BMI and the mPNNSGS, we still reported OR lower than 1 in each group (Fig. 2). 
Table 1. Characteristics of the participants, before imputation, according to the quintiles of total fibre intake, among women ( $n 26640)$ and men ( $n$ 8740) from the NutriNet-Santé study (Mean values and standard deviations; numbers and percentages)

\begin{tabular}{|c|c|c|c|c|c|c|c|c|c|c|c|c|c|c|c|c|c|c|c|c|}
\hline & \multicolumn{10}{|c|}{ Women (n 26 640) } & \multicolumn{10}{|c|}{ Men $(n 8740)$} \\
\hline & \multicolumn{2}{|c|}{ Quintile 1} & \multicolumn{2}{|c|}{ Quintile 2} & \multicolumn{2}{|c|}{ Quintile 3} & \multicolumn{2}{|c|}{ Quintile 4} & \multicolumn{2}{|c|}{ Quintile 5} & \multicolumn{2}{|c|}{ Quintile 1} & \multicolumn{2}{|c|}{ Quintile 2} & \multicolumn{2}{|c|}{ Quintile 3} & \multicolumn{2}{|c|}{ Quintile 4} & \multicolumn{2}{|c|}{ Quintile 5} \\
\hline & $\begin{array}{l}\text { Mean } \\
\text { or } n\end{array}$ & $\begin{array}{l}\text { SD or } \\
\%\end{array}$ & $\begin{array}{l}\text { Mean } \\
\text { or } n\end{array}$ & $\begin{array}{l}\text { SD or } \\
\%\end{array}$ & $\begin{array}{l}\text { Mean } \\
\text { or } n\end{array}$ & $\begin{array}{l}\text { SD or } \\
\%\end{array}$ & $\begin{array}{l}\text { Mean } \\
\text { or } n\end{array}$ & $\begin{array}{c}\text { SD or } \\
\%\end{array}$ & $\begin{array}{l}\text { Mean } \\
\text { or } n\end{array}$ & $\begin{array}{c}\text { SD or } \\
\%\end{array}$ & $\begin{array}{l}\text { Mean } \\
\text { or } n\end{array}$ & $\begin{array}{c}\text { SD or } \\
\%\end{array}$ & $\begin{array}{l}\text { Mean } \\
\text { or } n\end{array}$ & $\begin{array}{l}\text { SD or } \\
\%\end{array}$ & $\begin{array}{l}\text { Mean } \\
\text { or } n\end{array}$ & $\begin{array}{l}\text { SD or } \\
\%\end{array}$ & $\begin{array}{l}\text { Mean } \\
\text { or } n\end{array}$ & $\begin{array}{l}\text { SD or } \\
\%\end{array}$ & $\begin{array}{l}\text { Mean } \\
\text { or } n\end{array}$ & $\begin{array}{c}\text { SD or } \\
\%\end{array}$ \\
\hline Age (years) (mean and SD) & $45 \cdot 1$ & $18 \cdot 9$ & $50 \cdot 8$ & 13.4 & 53.9 & $13 \cdot 1$ & $56 \cdot 5$ & $12 \cdot 6$ & $56 \cdot 6$ & 13.0 & $52 \cdot 6$ & $14 \cdot 0$ & $58 \cdot 0$ & $13 \cdot 3$ & 61.5 & $12 \cdot 8$ & $62 \cdot 3$ & $12 \cdot 0$ & $62 \cdot 1$ & $12 \cdot 3$ \\
\hline \multicolumn{21}{|l|}{ Smoking ( $n$ and \%) } \\
\hline Never smokers & 2855 & 53.6 & 2839 & 53.3 & 2928 & $55 \cdot 0$ & 2932 & $55 \cdot 0$ & 2907 & 54.6 & 765 & 43.8 & 690 & 39.5 & 673 & 38.5 & 708 & $40 \cdot 5$ & 770 & 44.1 \\
\hline Former smokers & 1470 & $27 \cdot 6$ & 1749 & $32 \cdot 8$ & 1799 & 33.8 & 1937 & $36 \cdot 4$ & 2008 & 37.7 & 649 & $37 \cdot 1$ & 832 & $47 \cdot 6$ & 894 & $51 \cdot 1$ & 899 & 51.4 & 862 & $49 \cdot 3$ \\
\hline Current smokers & 1003 & $18 \cdot 8$ & 740 & 13.9 & 601 & $11 \cdot 2$ & 459 & 8.6 & 412 & $7 \cdot 7$ & 334 & $19 \cdot 1$ & 226 & $12 \cdot 9$ & 181 & $10 \cdot 4$ & 141 & $8 \cdot 1$ & 116 & $6 \cdot 6$ \\
\hline Missing & 0 & 0.0 & 0 & 0.0 & 0 & 0.0 & 0 & 0.0 & 1 & 0.0 & 0 & 0.0 & 0 & 0.0 & 0 & 0.0 & 0 & 0.0 & 0 & 0.0 \\
\hline $\begin{array}{l}\text { Pack years among ever smokers } \\
\text { (mean and } \mathrm{SD})\end{array}$ & $11 \cdot 2$ & $12 \cdot 1$ & $11 \cdot 6$ & 13.5 & $11 \cdot 2$ & $12 \cdot 9$ & $11 \cdot 1$ & $12 \cdot 3$ & 11.4 & $13 \cdot 4$ & $19 \cdot 2$ & $19 \cdot 1$ & $17 \cdot 3$ & $16 \cdot 3$ & $16 \cdot 9$ & $15 \cdot 4$ & $15 \cdot 6$ & $14 \cdot 9$ & $15 \cdot 2$ & $15 \cdot 3$ \\
\hline \multicolumn{21}{|l|}{ Educational level ( $n$ and \%) } \\
\hline$\leq 13$ & 827 & $15 \cdot 5$ & 935 & $17 \cdot 6$ & 949 & $17 \cdot 8$ & 973 & $18 \cdot 3$ & 893 & $16 \cdot 8$ & 425 & $24 \cdot 3$ & 456 & $26 \cdot 1$ & 438 & $25 \cdot 1$ & 450 & $25 \cdot 7$ & 398 & $22 \cdot 8$ \\
\hline 14 & 993 & $18 \cdot 6$ & 852 & $16 \cdot 0$ & 786 & $14 \cdot 8$ & 816 & $15 \cdot 3$ & 792 & 14.9 & 245 & 14.0 & 216 & $12 \cdot 4$ & 218 & 12.5 & 198 & $11 \cdot 3$ & 190 & $10 \cdot 9$ \\
\hline $15-16$ & 1776 & 33.3 & 1704 & $32 \cdot 0$ & 1761 & 33.1 & 1718 & $32 \cdot 2$ & 1729 & 32.5 & 480 & 27.5 & 403 & 23.1 & 379 & 21.7 & 384 & $22 \cdot 0$ & 420 & 24.0 \\
\hline$\geq 17$ & 1695 & 31.8 & 1802 & 33.8 & 1794 & 33.7 & 1780 & 33.4 & 1872 & $35 \cdot 1$ & 593 & 33.9 & 666 & $38 \cdot 1$ & 703 & $40 \cdot 2$ & 708 & 40.5 & 729 & 41.7 \\
\hline Missing & 37 & 0.7 & 35 & 0.7 & 38 & 0.7 & 41 & 0.8 & 42 & 0.8 & 5 & 0.3 & 7 & 0.4 & 10 & 0.6 & 8 & 0.5 & 11 & 0.6 \\
\hline \multicolumn{21}{|l|}{ Leisure-time physical activity ( $n$ and \%) } \\
\hline High & 1145 & 21.5 & 1324 & 24.9 & 1489 & $28 \cdot 0$ & 1684 & 31.6 & 1981 & $37 \cdot 2$ & 570 & $32 \cdot 6$ & 641 & $36 \cdot 7$ & 675 & $38 \cdot 6$ & 735 & $42 \cdot 1$ & 790 & $45 \cdot 2$ \\
\hline Moderate & 1999 & 37.5 & 2143 & $40 \cdot 2$ & 2200 & 41.3 & 2133 & $40 \cdot 0$ & 2071 & 38.9 & 546 & $31 \cdot 2$ & 592 & 33.9 & 600 & $34 \cdot 3$ & 604 & 34.6 & 613 & $35 \cdot 1$ \\
\hline Low & 1442 & $27 \cdot 1$ & 1207 & $22 \cdot 7$ & 981 & $18 \cdot 4$ & 929 & $17 \cdot 4$ & 758 & $14 \cdot 2$ & 462 & $26 \cdot 4$ & 340 & 19.5 & 299 & $17 \cdot 1$ & 248 & $14 \cdot 2$ & 199 & 11.4 \\
\hline Missing & 742 & 13.9 & 654 & $12 \cdot 3$ & 658 & $12 \cdot 4$ & 582 & $10 \cdot 9$ & 518 & $9 \cdot 7$ & 170 & 9.7 & 175 & $10 \cdot 0$ & 174 & $10 \cdot 0$ & 161 & $9 \cdot 2$ & 146 & 8.4 \\
\hline Total daily energy (mean and $\mathrm{SD}$ ) & 1868 & 409 & 1759 & 376 & 1741 & 367 & 1737 & 360 & 1792 & 377 & 2364 & 519 & 2215 & 468 & 2198 & 469 & 2213 & 479 & 2312 & 492 \\
\hline $\mathrm{BMI}\left(\mathrm{kg} / \mathrm{m}^{2}\right)$ (mean and $\left.\mathrm{sD}\right)$ & 23.8 & $5 \cdot 0$ & 23.7 & 4.5 & 23.6 & $4 \cdot 3$ & 23.5 & $4 \cdot 2$ & $22 \cdot 8$ & $4 \cdot 2$ & $25 \cdot 4$ & $4 \cdot 2$ & $25 \cdot 3$ & 3.8 & $25 \cdot 3$ & 3.8 & 24.9 & 3.4 & $24 \cdot 1$ & 3.3 \\
\hline \multicolumn{21}{|l|}{$\mathrm{BMI}\left(\mathrm{kg} / \mathrm{m}^{2}\right)(n$ and \%) } \\
\hline$<18.5$ & 322 & $6 \cdot 0$ & 255 & $4 \cdot 8$ & 256 & $4 \cdot 8$ & 249 & 4.7 & 450 & 8.5 & 23 & $1 \cdot 3$ & 17 & 1.0 & 16 & 0.9 & 11 & 0.6 & 17 & $1 \cdot 0$ \\
\hline $18.5-24.9$ & 3377 & 63.4 & 3429 & 64.4 & 3529 & $66 \cdot 2$ & 3560 & $66 \cdot 8$ & 3645 & 68.4 & 871 & $49 \cdot 8$ & 899 & 51.4 & 907 & 51.9 & 978 & $56 \cdot 0$ & 1144 & 65.5 \\
\hline $25 \cdot 0-29.9$ & 937 & $17 \cdot 6$ & 1081 & $20 \cdot 3$ & 1064 & $20 \cdot 0$ & 1049 & $19 \cdot 7$ & 872 & $16 \cdot 4$ & 661 & 37.8 & 658 & $37 \cdot 6$ & 644 & $36 \cdot 8$ & 626 & $35 \cdot 8$ & 487 & $27 \cdot 9$ \\
\hline$\geq 30 \cdot 0$ & 579 & 10.9 & 485 & $9 \cdot 1$ & 426 & 8.0 & 416 & 7.8 & 323 & $6 \cdot 1$ & 193 & 11.0 & 173 & 9.9 & 180 & $10 \cdot 3$ & 133 & 7.6 & 96 & 5.5 \\
\hline Missing & 113 & $2 \cdot 1$ & 78 & 1.5 & 53 & $1 \cdot 0$ & 54 & 1.0 & 38 & 0.7 & 0 & 0.0 & 1 & 0.1 & 1 & 0.1 & 0 & 0.0 & 4 & 0.2 \\
\hline Allergic rhinitis ( $n$ and \%) & 2447 & $45 \cdot 9$ & 2313 & 43.4 & 2257 & $42 \cdot 4$ & 2076 & 39.0 & 2128 & 39.9 & 499 & $28 \cdot 6$ & 541 & 31.0 & 519 & $29 \cdot 7$ & 538 & $30 \cdot 8$ & 508 & 29.1 \\
\hline Family history of asthma ( $n$ and \%) & 540 & $10 \cdot 1$ & 540 & $10 \cdot 1$ & 565 & $10 \cdot 6$ & 557 & 10.5 & 548 & $10 \cdot 3$ & 139 & 8.0 & 119 & 6.8 & 130 & $7 \cdot 4$ & 127 & 7.3 & 124 & $7 \cdot 1$ \\
\hline
\end{tabular}


Table 1. (Continued)

\begin{tabular}{|c|c|c|c|c|c|c|c|c|c|c|c|c|c|c|c|c|c|c|c|c|}
\hline & \multicolumn{10}{|c|}{ Women (n 26 640) } & \multicolumn{10}{|c|}{ Men $(n 8740)$} \\
\hline & \multicolumn{2}{|c|}{ Quintile 1} & \multicolumn{2}{|c|}{ Quintile 2} & \multicolumn{2}{|c|}{ Quintile 3} & \multicolumn{2}{|c|}{ Quintile 4} & \multicolumn{2}{|c|}{ Quintile 5} & \multicolumn{2}{|c|}{ Quintile 1} & \multicolumn{2}{|c|}{ Quintile 2} & \multicolumn{2}{|c|}{ Quintile 3} & \multicolumn{2}{|c|}{ Quintile 4} & \multicolumn{2}{|c|}{ Quintile 5} \\
\hline & $\begin{array}{l}\text { Mean } \\
\text { or } n\end{array}$ & $\begin{array}{c}\text { SD or } \\
\%\end{array}$ & $\begin{array}{c}\text { Mean } \\
\text { or } n\end{array}$ & $\begin{array}{c}\text { SD or } \\
\%\end{array}$ & $\begin{array}{c}\text { Mean } \\
\text { or } n\end{array}$ & $\begin{array}{c}\text { SD or } \\
\%\end{array}$ & $\begin{array}{c}\text { Mean } \\
\text { or } n\end{array}$ & $\begin{array}{c}\text { SD or } \\
\%\end{array}$ & $\begin{array}{l}\text { Mean } \\
\text { or } n\end{array}$ & $\begin{array}{c}\text { SD or } \\
\%\end{array}$ & $\begin{array}{c}\text { Mean } \\
\text { or } n\end{array}$ & $\begin{array}{c}\text { SD or } \\
\%\end{array}$ & $\begin{array}{c}\text { Mean } \\
\text { or } n\end{array}$ & $\begin{array}{c}\text { SD or } \\
\%\end{array}$ & $\begin{array}{c}\text { Mean } \\
\text { or } n\end{array}$ & $\begin{array}{c}\text { SD or } \\
\%\end{array}$ & $\begin{array}{c}\text { Mean } \\
\text { or } n\end{array}$ & $\begin{array}{c}\text { SD or } \\
\%\end{array}$ & $\begin{array}{c}\text { Mean } \\
\text { or } n\end{array}$ & $\begin{array}{c}\text { SD or } \\
\%\end{array}$ \\
\hline Asthma symptom score ${ }^{*}$ (mean and sD) & 0.50 & 0.90 & 0.43 & 0.82 & 0.42 & 0.82 & 0.39 & 0.81 & 0.36 & 0.75 & 0.47 & 0.89 & 0.40 & 0.80 & 0.39 & 0.81 & 0.36 & 0.79 & 0.28 & 0.66 \\
\hline $0(n$ and \%) & 3626 & $68 \cdot 1$ & 3761 & $70 \cdot 6$ & 3849 & $72 \cdot 2$ & 3940 & $74 \cdot 0$ & 4015 & 75.4 & 1226 & $70 \cdot 1$ & 1289 & 73.7 & 1311 & $75 \cdot 0$ & 1332 & $76 \cdot 2$ & 1405 & 80.4 \\
\hline $1(n$ and $\%)$ & 1118 & 21.0 & 1100 & $20 \cdot 7$ & 1011 & $19 \cdot 0$ & 973 & $18 \cdot 3$ & 933 & 17.5 & 345 & $19 \cdot 7$ & 310 & $17 \cdot 7$ & 292 & $16 \cdot 7$ & 280 & $16 \cdot 0$ & 247 & $14 \cdot 1$ \\
\hline $2-5(n$ and \%) & 584 & $11 \cdot 0$ & 467 & $8 \cdot 8$ & 468 & $8 \cdot 8$ & 415 & $7 \cdot 8$ & 380 & $7 \cdot 1$ & 177 & $10 \cdot 1$ & 149 & 8.5 & 145 & $8 \cdot 3$ & 136 & 7.8 & 96 & $5 \cdot 5$ \\
\hline Ever asthma† ( $n$ and \%) & 749 & $14 \cdot 1$ & 638 & $12 \cdot 0$ & 639 & $12 \cdot 0$ & 561 & $10 \cdot 5$ & 510 & $9 \cdot 6$ & 216 & $12 \cdot 4$ & 157 & $9 \cdot 0$ & 163 & $9 \cdot 3$ & 154 & $8 \cdot 8$ & 152 & $8 \cdot 7$ \\
\hline \multicolumn{21}{|l|}{ Asthma control test ( $n$ and \%) } \\
\hline$>19$ & 438 & $85 \cdot 7$ & 348 & $82 \cdot 1$ & 365 & $85 \cdot 7$ & 318 & $84 \cdot 8$ & 321 & $89 \cdot 7$ & 116 & $84 \cdot 7$ & 89 & $88 \cdot 1$ & 100 & $90 \cdot 1$ & 97 & $89 \cdot 8$ & 93 & $93 \cdot 0$ \\
\hline$\leq 19$ & 73 & $14 \cdot 3$ & 76 & $17 \cdot 9$ & 61 & $14 \cdot 3$ & 57 & $15 \cdot 2$ & 37 & $10 \cdot 3$ & 21 & $15 \cdot 3$ & 12 & 11.9 & 11 & 9.9 & 11 & $10 \cdot 2$ & 7 & $7 \cdot 0$ \\
\hline \multicolumn{21}{|l|}{ Fibre intake (g/d) (mean and sD) } \\
\hline Total & $13 \cdot 8$ & 1.7 & $17 \cdot 2$ & 0.7 & $19 \cdot 6$ & 0.7 & $22 \cdot 4$ & 0.9 & 28.6 & 4.9 & $12 \cdot 7$ & $2 \cdot 1$ & $16 \cdot 8$ & 0.8 & $19 \cdot 6$ & 0.8 & $22 \cdot 9$ & $1 \cdot 1$ & 30.5 & $5 \cdot 7$ \\
\hline Soluble & 3.8 & $1 \cdot 0$ & 4.9 & 0.9 & $5 \cdot 8$ & 1.0 & $6 \cdot 7$ & $1 \cdot 1$ & 8.6 & $2 \cdot 2$ & 3.6 & $1 \cdot 3$ & $5 \cdot 0$ & $1 \cdot 2$ & $5 \cdot 9$ & 1.4 & $7 \cdot 0$ & 1.5 & 9.5 & $2 \cdot 8$ \\
\hline Insoluble & $10 \cdot 0$ & 1.4 & $12 \cdot 3$ & 1.0 & $13 \cdot 8$ & $1 \cdot 1$ & $15 \cdot 7$ & 1.3 & $20 \cdot 0$ & 3.7 & $9 \cdot 1$ & 1.8 & 11.8 & 1.3 & 13.7 & 1.5 & $15 \cdot 9$ & 1.7 & 21.0 & $4 \cdot 1$ \\
\hline Cereal & $4 \cdot 3$ & 1.5 & $5 \cdot 1$ & 1.5 & $5 \cdot 7$ & 1.7 & $6 \cdot 3$ & $2 \cdot 0$ & 7.5 & 3.0 & $4 \cdot 2$ & 1.9 & 5.4 & 1.9 & $6 \cdot 2$ & $2 \cdot 2$ & $7 \cdot 1$ & $2 \cdot 6$ & $9 \cdot 2$ & $4 \cdot 3$ \\
\hline Vegetable & $3 \cdot 1$ & $1 \cdot 3$ & $4 \cdot 3$ & 1.4 & $5 \cdot 1$ & 1.6 & 5.9 & 1.8 & $7 \cdot 6$ & 3.2 & 3.0 & 1.5 & $4 \cdot 2$ & 1.6 & $5 \cdot 0$ & 1.8 & 5.9 & $2 \cdot 1$ & 7.5 & $3 \cdot 2$ \\
\hline Fruit & 1.9 & 1.2 & 3.0 & 1.4 & 3.8 & 1.6 & 4.8 & 1.9 & 6.5 & 3.3 & 1.5 & 1.4 & 2.9 & 1.7 & 3.9 & $2 \cdot 0$ & 4.9 & $2 \cdot 4$ & $7 \cdot 0$ & 4.0 \\
\hline Seed & 0.2 & 0.4 & 0.3 & 0.5 & 0.4 & 0.6 & 0.5 & 0.8 & 0.9 & $1 \cdot 3$ & $0 \cdot 1$ & 0.5 & 0.3 & 0.6 & 0.3 & 0.7 & 0.4 & $0 \cdot 8$ & 1.0 & 1.9 \\
\hline Legume & 0.3 & 0.6 & 0.5 & 0.7 & 0.6 & 0.8 & 0.8 & 1.0 & $1 \cdot 3$ & $1 \cdot 7$ & 0.3 & 0.7 & 0.5 & 0.9 & 0.6 & $1 \cdot 0$ & 0.8 & $1 \cdot 2$ & 1.5 & $2 \cdot 2$ \\
\hline mPNNS-GS (mean and sD) & $7 \cdot 0$ & 1.3 & 7.8 & 1.2 & 8.3 & 1.3 & 8.8 & 1.3 & 9.5 & 1.4 & $6 \cdot 7$ & 1.3 & 7.6 & 1.3 & $8 \cdot 1$ & 1.3 & 8.7 & 1.3 & 9.2 & 1.4 \\
\hline
\end{tabular}

mPNNS-GS, Modified Programme National Nutrition Santé guidelines score. mPNNS-GS, Modified Programme National Nutrition Santé guidelines score.
${ }^{*}$ Number of respiratory symptoms during the past 12 months: (1) breathless while wheezing, (2) woken up with chest tightness, (3) attack of shortness of breath at rest, (4) attack of shortness of breath after exercise and (5) woken by attack of
shortness of breath. Each item is scored from 0 to 1 , and the total asthma symptom score ranges from 0 to 5 .

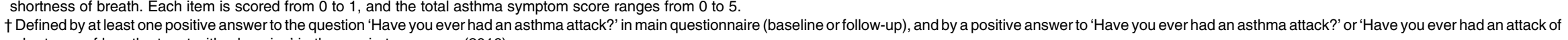
shorness

‡ Among 2063 women and 546 men. Based on five questions in the last 4 weeks on: (1) activity limitations: asthma keep from getting as much done at work, school or at home, 'some of the time' to 'all of the time'; (2) shortness of breath: '3-6 times/week' to 'more than once daily'; (3) woken up by asthma symptoms at night: 'once/week' to 'every night'; (4) use $\beta$-agonist inhaler '2 times/week' to ' $3+$ times daily' and (5) self-rated asthma control: 'somewhat controlled' to 'not controlled at all'. Each item is scored from 1 to 5 , and the total asthma control test score ranges from 5 to 25 . 
Table 2. Association between quintiles of dietary fibre intake and the asthma symptom score (continuous variable) among women from the NutriNet-Santé study ( $n 26$ 640)

(Odds ratios and $95 \%$ confidence intervals)

\begin{tabular}{|c|c|c|c|c|c|c|c|c|c|c|c|}
\hline & \multicolumn{2}{|c|}{ Quintile 1} & \multicolumn{2}{|c|}{ Quintile 2} & \multicolumn{2}{|c|}{ Quintile 3} & \multicolumn{2}{|c|}{ Quintile 4} & \multicolumn{2}{|c|}{ Quintile 5} & \multirow[b]{2}{*}{$P$ for trend } \\
\hline & OR & $95 \% \mathrm{Cl}$ & OR & $95 \% \mathrm{Cl}$ & OR & $95 \% \mathrm{Cl}$ & OR & $95 \% \mathrm{Cl}$ & OR & $95 \% \mathrm{Cl}$ & \\
\hline$n$ & \multicolumn{2}{|c|}{5328} & \multicolumn{2}{|c|}{5328} & \multicolumn{2}{|c|}{5328} & \multicolumn{2}{|c|}{5328} & \multicolumn{2}{|c|}{5328} & \\
\hline \multirow[t]{2}{*}{ Total fibre } & $1.00^{*}$ & Ref & $0.85^{*}$ & $0.79,0.92$ & $0.82^{*}$ & $0.76,0.88$ & $0.76^{*}$ & $0.70,0.82$ & $0.70^{\star}$ & $0.64,0.75$ & $<0.0001$ \\
\hline & $1.00 \dagger$ & Ref & $0.87 \dagger$ & $0.81,0.93$ & $0.84 \dagger$ & $0.78,0.90$ & $0.80 \dagger$ & $0.74,0.86$ & $0.73 \dagger$ & $0.67,0.79$ & $<0.0001$ \\
\hline \multirow[t]{2}{*}{ Soluble fibre } & $1.00^{*}$ & Ref & $0.79^{*}$ & $0.74,0.85$ & $0.78^{*}$ & $0.73,0.84$ & $0.73^{*}$ & $0.68,0.79$ & $0.65^{\star}$ & $0.60,0.71$ & $<0.0001$ \\
\hline & $1.00 \dagger$ & Ref & $0.81 \dagger$ & $0.76,0.88$ & $0.82 \dagger$ & $0.76,0.88$ & $0.77 \dagger$ & $0.72,0.83$ & $0.71 \dagger$ & $0.65,0.76$ & $<0.0001$ \\
\hline \multirow[t]{2}{*}{ Insoluble fibre } & $1.00^{*}$ & Ref & $0.89^{*}$ & $0.83,0.96$ & $0.85^{*}$ & $0.79,0.92$ & $0.81^{*}$ & $0.76,0.88$ & $0.74^{*}$ & $0.69,0.80$ & $<0.0001$ \\
\hline & $1.00 \dagger$ & Ref & $0.91 \dagger$ & $0.85,0.98$ & $0.87 \dagger$ & $0.81,0.94$ & $0.85 \dagger$ & $0.79,0.92$ & $0.77 \dagger$ & $0.71,0.83$ & $<0.0001$ \\
\hline \multirow[t]{2}{*}{ Cereal fibre } & $1.00^{*}$ & Ref & $0.85^{*}$ & $0.81,0.93$ & $0.80^{*}$ & $0.74,0.86$ & $0.80^{*}$ & $0.74,0.86$ & $0.76^{*}$ & $0.70,0.82$ & $<0.0001$ \\
\hline & $1.00 \dagger$ & Ref & $0.88 \dagger$ & $0.82,0.94$ & $0.82 \dagger$ & $0.76,0.88$ & $0.83 \dagger$ & $0.77,0.89$ & $0.81 \dagger$ & $0.75,0.87$ & $<0.0001$ \\
\hline \multirow[t]{2}{*}{ Vegetable fibre } & $1.00^{*}$ & Ref & $0.86^{*}$ & $0.80,0.92$ & $0.86^{*}$ & $0.80,0.93$ & $0.86^{*}$ & $0.80,0.92$ & $0.80^{*}$ & $0.74,0.86$ & $<0.0001$ \\
\hline & $1.00 \dagger$ & Ref & $0.87 \dagger$ & $0.81,0.94$ & $0.87 \dagger$ & $0.81,0.94$ & $0.88 \dagger$ & $0.82,0.95$ & $0.82 \dagger$ & $0.76,0.89$ & $<0.0001$ \\
\hline \multirow[t]{2}{*}{ Fruit fibre } & $1.00^{*}$ & Ref & $0.89^{*}$ & $0.82,0.95$ & $0.83^{*}$ & $0.77,0.88$ & $0.76^{*}$ & $0.71,0.82$ & $0.68^{*}$ & $0.63,0.74$ & $<0.0001$ \\
\hline & $1.00 \dagger$ & Ref & $0.92 \dagger$ & $0.86,0.99$ & $0.88 \dagger$ & $0.82,0.95$ & $0.81 \dagger$ & $0.75,0.88$ & $0.74 \dagger$ & $0.69,0.80$ & $<0.0001$ \\
\hline \multirow[t]{2}{*}{ Seed fibre } & $1.00^{*}$ & Ref & $0.94^{*}$ & $0.88,1.02$ & $0.95^{*}$ & $0.88,1.02$ & $0.88^{\star}$ & $0.81,0.95$ & $0.81^{\star}$ & $0.75,0.87$ & $<0.0001$ \\
\hline & $1.00 \dagger$ & Ref & $0.96 \dagger$ & $0.89,1.04$ & $0.95 \dagger$ & $0.88,1.03$ & $0.89 \dagger$ & $0.83,0.97$ & $0.81 \dagger$ & $0.75,0.88$ & $<0.0001$ \\
\hline \multirow[t]{2}{*}{ Legume fibre } & $1.00^{*}$ & Ref & $0.92^{*}$ & $0.85,0.99$ & $1.00^{*}$ & $0.92,1.07$ & $0.93^{*}$ & $0.87,1.00$ & $0.92^{*}$ & $0.85,0.99$ & 0.06 \\
\hline & $1.00 \dagger$ & Ref & $0.97 \dagger$ & $0.89,1.05$ & $1.02 \dagger$ & $0.94,1.11$ & $0.96 \dagger$ & $0.89,1.04$ & $0.94 \dagger$ & $0.87,1.01$ & 0.06 \\
\hline
\end{tabular}

Ref, reference.

* Adjusted for age.

† Further adjusted for smoking, pack-years (among ever smokers), educational level, leisure-time physical activity, total daily energy, allergic rhinitis and family history of asthma.

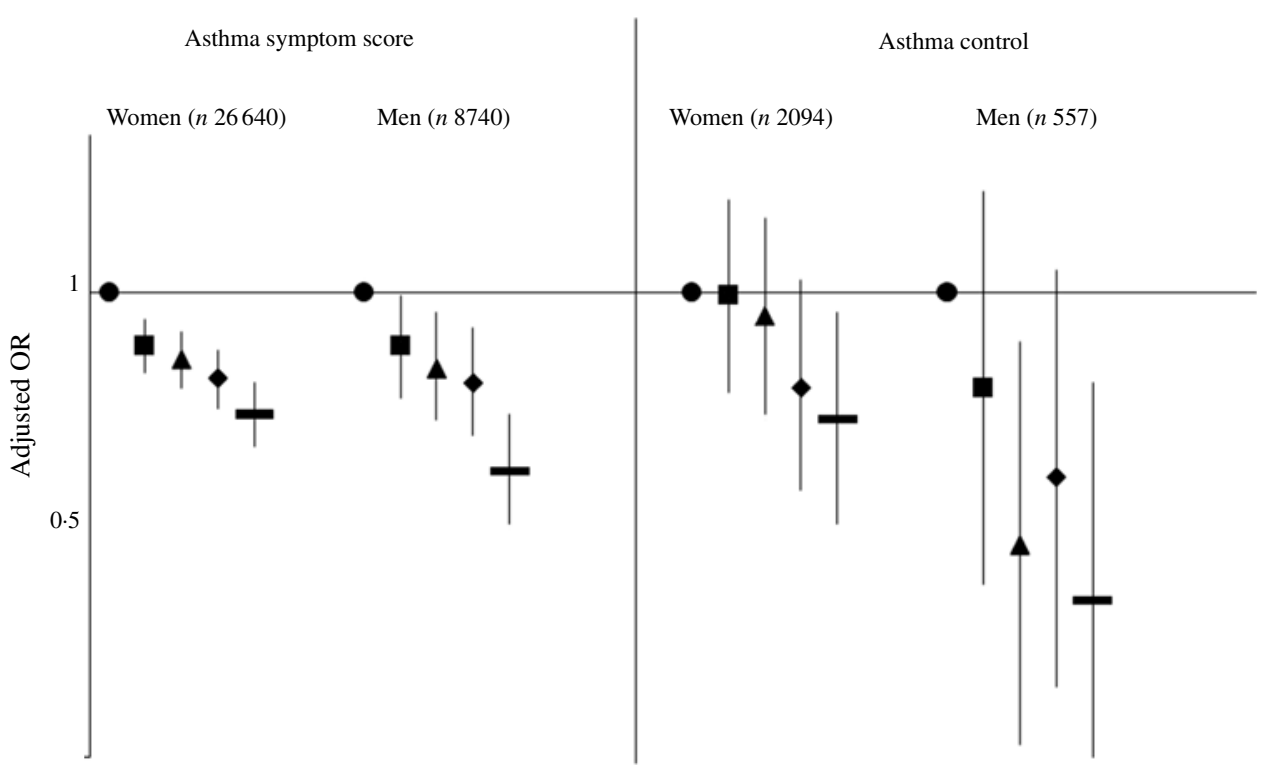

Fig. 1. Associations between quintiles of total dietary fibre intake with the asthma symptom score (continuous) and the asthma control (controlled $v$. uncontrolled) among women and men. Values are adjusted odds ratios and $95 \%$ confidence intervals.

For smoking, we observed association of similar magnitude in never, former and current smokers, especially among women (Fig. 2). The interaction between total dietary fibre intake with smoking status was not statistically significant $(P=0.66$ in women and 0.39 in men). For BMI, the association between total dietary fibre intake and asthma symptom was significant only among participants with BMI $<30.0 \mathrm{~kg} / \mathrm{m}^{2}$, but we reported no significant interaction between total dietary fibre and BMI ( $P=0.13$ in women and 0.36 in men). For the mPNNS-GS, we observed similar association between total dietary fibre intake and asthma symptom in each tertile, with no significant interaction between mPNNS-GS and total dietary fibre ( $P=0.19$ in women and 0.29 in men). Finally, when analyses were restricted to participants without ever asthma ( $n 24159$ women and 8069 men), we found similar associations between dietary fibre intake and the asthma symptom score: OR for the participants in the highest quintile of total dietary fibre was 0.74 (95\% CI $0.68,0.81$ ) in women and 0.68 (95\% CI 0.58 , $0.79)$ in men, as compared with participants in the lowest quintile.

When looking at the type of fibre (soluble or insoluble), we observed a significant inverse association of similar magnitude 
Table 3. Associations between quintiles of dietary fibre intake and the asthma symptom score (continuous variable) among men from the NutriNet-Santé study $(n$ 8740)

(Odds ratios and $95 \%$ confidence intervals)

\begin{tabular}{|c|c|c|c|c|c|c|c|c|c|c|c|}
\hline & \multicolumn{2}{|c|}{ Quintile 1} & \multicolumn{2}{|c|}{ Quintile 2} & \multicolumn{2}{|c|}{ Quintile 3} & \multicolumn{2}{|c|}{ Quintile 4} & \multicolumn{2}{|c|}{ Quintile 5} & \multirow[b]{2}{*}{$P$ for trend } \\
\hline & OR & $95 \% \mathrm{Cl}$ & OR & $95 \% \mathrm{Cl}$ & OR & $95 \% \mathrm{Cl}$ & OR & $95 \% \mathrm{Cl}$ & OR & $95 \% \mathrm{Cl}$ & \\
\hline$n$ & \multicolumn{2}{|c|}{1748} & \multicolumn{2}{|c|}{1748} & \multicolumn{2}{|c|}{1748} & \multicolumn{2}{|c|}{1748} & \multicolumn{2}{|c|}{1748} & \\
\hline \multirow[t]{2}{*}{ Total fibre } & $1.00^{*}$ & Ref & $0.83^{*}$ & $0.72,0.95$ & $0.79^{*}$ & $0.69,0.91$ & $0.74^{*}$ & $0.65,0.86$ & $0.57^{*}$ & $0.49,0.66$ & $<0.0001$ \\
\hline & $1.00 \dagger$ & Ref & $0.87 \dagger$ & $0.76,0.99$ & $0.82 \dagger$ & $0.72,0.95$ & $0.79 \dagger$ & $0.69,0.91$ & $0.63 \dagger$ & $0.55,0.73$ & $<0.0001$ \\
\hline \multirow[t]{2}{*}{ Soluble fibre } & $1.00^{*}$ & Ref & $0.87^{*}$ & $0.76,0.99$ & $0.78^{*}$ & $0.68,0.90$ & $0.75^{*}$ & $0.65,0.86$ & $0.56^{*}$ & $0.48,0.65$ & $<0.0001$ \\
\hline & $1.00 \dagger$ & Ref & $0.89 \dagger$ & $0.78,1.01$ & $0.83 \dagger$ & $0.73,0.95$ & $0.81 \dagger$ & $0.71,0.93$ & $0.63 \dagger$ & $0.54,0.73$ & $<0.0001$ \\
\hline \multirow[t]{2}{*}{ Insoluble fibre } & $1.00^{*}$ & Ref & $0.83^{*}$ & $0.73,0.96$ & $0.81^{*}$ & $0.71,0.94$ & $0.82^{*}$ & $0.72,0.95$ & $0.62^{*}$ & $0.54,0.72$ & $<0.0001$ \\
\hline & $1.00 \dagger$ & Ref & $0.88 \dagger$ & $0.77,1.00$ & $0.87 \dagger$ & $0.76,0.99$ & $0.83 \dagger$ & $0.72,0.95$ & $0.69 \dagger$ & $0.60,0.80$ & $<0.0001$ \\
\hline \multirow[t]{2}{*}{ Cereal fibre } & $1.00^{*}$ & Ref & $0.98^{*}$ & $0.86,1.12$ & $0.85^{*}$ & $0.74,0.97$ & $0.83^{*}$ & $0.72,0.96$ & $0.68^{*}$ & $0.59,0.79$ & $<0.0001$ \\
\hline & $1.00 \dagger$ & Ref & $0.97 \dagger$ & $0.84,1 \cdot 10$ & $0.83 \dagger$ & $0.72,0.95$ & $0.84 \dagger$ & $0.73,0.96$ & $0.71 \dagger$ & $0.61,0.82$ & $<0.0001$ \\
\hline \multirow[t]{2}{*}{ Vegetable fibre } & $1.00^{*}$ & Ref & $0.85^{\star}$ & $0.74,0.98$ & $0.81^{*}$ & $0.71,0.94$ & $0.90^{*}$ & $0.78,1.03$ & $0.80^{*}$ & $0.69,0.92$ & 0.01 \\
\hline & $1.00 \dagger$ & Ref & $0.87 \dagger$ & $0.76,1.00$ & $0.82 \dagger$ & $0.72,0.94$ & $0.94 \dagger$ & $0.82,1.07$ & $0.84 \dagger$ & $0.73,0.97$ & 0.07 \\
\hline \multirow[t]{2}{*}{ Fruit fibre } & $1.00^{*}$ & Ref & $0.80^{*}$ & $0.70,0.92$ & $0.72^{*}$ & $0.63,0.83$ & $0.62^{*}$ & $0.53,0.71$ & $0.59^{*}$ & $0.51,0.68$ & $<0.0001$ \\
\hline & $1.00 \dagger$ & Ref & $0.80 \dagger$ & $0.70,0.91$ & $0.77 \dagger$ & $0.67,0.89$ & $0.69 \dagger$ & $0.60,0.80$ & $0.66 \dagger$ & $0.58,0.77$ & $<0.0001$ \\
\hline \multirow[t]{2}{*}{ Seed fibre } & $1.00^{*}$ & Ref & $1.09^{*}$ & $0.95,1.26$ & $1.08^{*}$ & $0.94,1.24$ & $1.12^{*}$ & $0.97,1.29$ & $0.86^{*}$ & $0.75,1.00$ & 0.004 \\
\hline & $1.00 \dagger$ & Ref & $1.09 \dagger$ & $0.95,1.26$ & $1.04 \dagger$ & $0.90,1.21$ & $1.11 \dagger$ & $0.96,1.28$ & $0.85 \dagger$ & $0.73,0.98$ & 0.002 \\
\hline \multirow[t]{2}{*}{ Legume fibre } & $1.00^{*}$ & Ref & $1.05^{*}$ & $0.91,1.20$ & $1.03^{*}$ & $0.89,1.18$ & $0.93^{*}$ & $0.81,1.08$ & $0.94^{*}$ & $0.81,1.08$ & 0.11 \\
\hline & $1.00 \dagger$ & Ref & $0.97 \dagger$ & $0.83,1.12$ & $0.96 \dagger$ & $0.83,1.12$ & $0.87 \dagger$ & $0.75,1.01$ & $0.89 \dagger$ & $0.77,1.03$ & 0.05 \\
\hline
\end{tabular}

Ref, reference.

${ }^{*}$ Adjusted for age.

† Further adjusted for smoking, pack-years (among ever smokers), educational level, leisure-time physical activity, total daily energy, allergic rhinitis and family history of asthma.

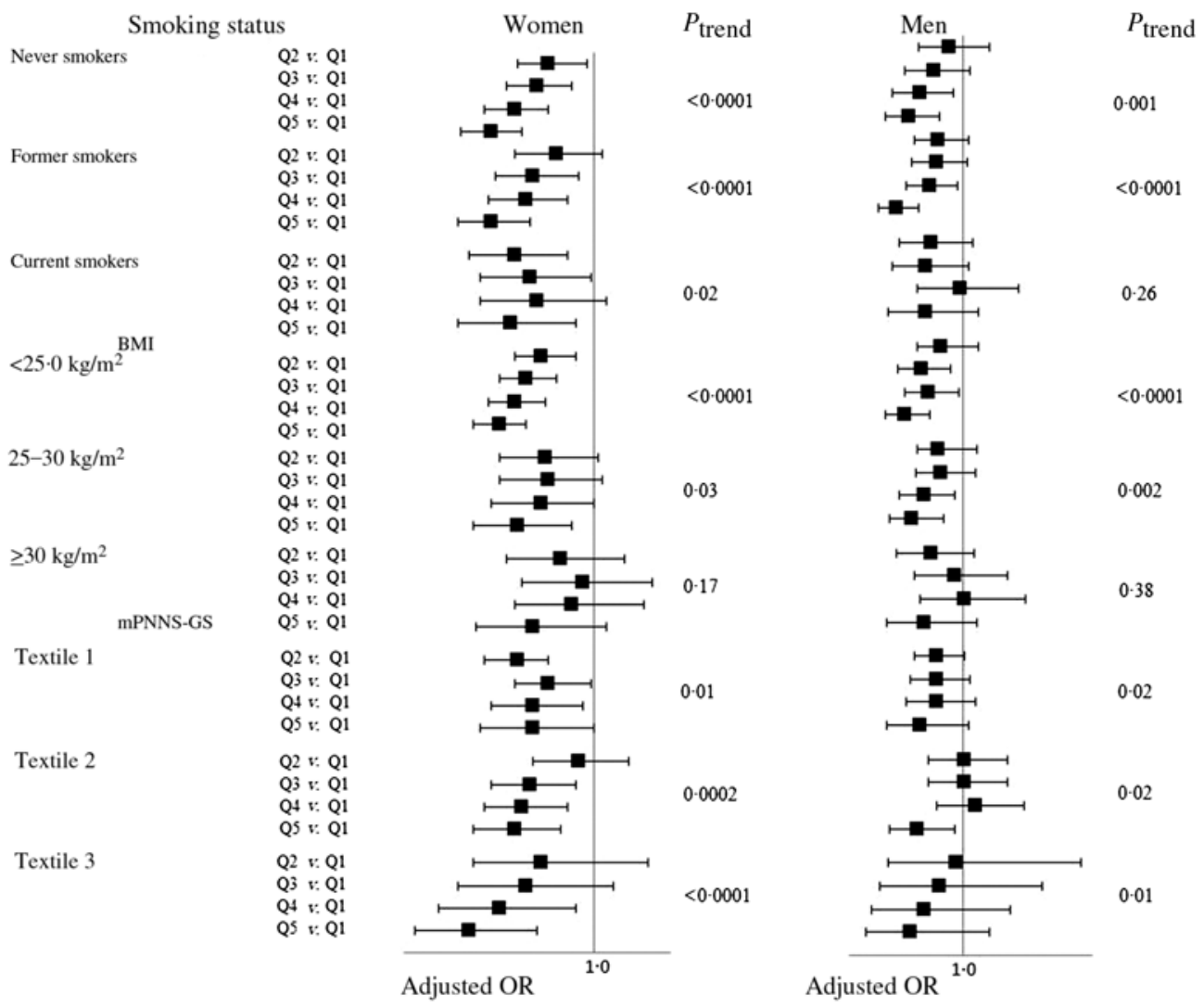

Fig. 2. Associations between quintiles $(\mathrm{Q})$ of total dietary fibre intake and the asthma symptom score (continuous variable) among women and men from the NutriNet-Santé study, stratified according to smoking, BMI and modified Programme National Nutrition Santé-guidelines score (mPNNS-GS). Models were adjusted for age, smoking (when appropriated), pack-years (among ever smokers), educational level, leisure-time physical activity, total daily energy, allergic rhinitis and family history of asthma. Values are adjusted odds ratios and $95 \%$ confidence intervals. 
Table 4. Association between quintiles of dietary fibre intake and the asthma control test score among women from the NutriNet-Santé study ( $n$ 2094) (Odds ratios and $95 \%$ confidence intervals)

\begin{tabular}{|c|c|c|c|c|c|c|c|c|c|c|c|}
\hline & \multicolumn{2}{|c|}{ Quintile 1} & \multicolumn{2}{|c|}{ Quintile 2} & \multicolumn{2}{|c|}{ Quintile 3} & \multicolumn{2}{|c|}{ Quintile 4} & \multicolumn{2}{|c|}{ Quintile 5} & \multirow[b]{2}{*}{$P$ for treno } \\
\hline & OR & $95 \% \mathrm{Cl}$ & OR & $95 \% \mathrm{Cl}$ & OR & $95 \% \mathrm{Cl}$ & OR & $95 \% \mathrm{Cl}$ & OR & $95 \% \mathrm{Cl}$ & \\
\hline \multicolumn{12}{|c|}{ Total fibre } \\
\hline$n$ & \multicolumn{2}{|c|}{511} & \multicolumn{2}{|c|}{424} & \multicolumn{2}{|c|}{426} & \multicolumn{2}{|c|}{375} & \multicolumn{2}{|c|}{358} & \\
\hline & $1.00^{*}$ & Ref & $1 \cdot 18^{\star}$ & $0.83,1.69$ & $0.83^{*}$ & $0.57,1.21$ & $0.86^{*}$ & $0.58,1.27$ & $0.54^{*}$ & $0.35,0.84$ & 0.003 \\
\hline & $1.00 \dagger$ & Ref & $1.00 \dagger$ & $0.78,1 \cdot 28$ & $0.93 \dagger$ & $0.72,1 \cdot 19$ & $0.79 \dagger$ & $0.60,1.02$ & $0.71 \dagger$ & $0.54,0.93$ & 0.003 \\
\hline & $1.00 \ddagger$ & Ref & $0.99 \ddagger$ & $0.77,1.27$ & $0.94 \ddagger$ & $0.73,1.21$ & $0.78 \ddagger$ & $0.60,1.03$ & $0.72 \ddagger$ & $0.55,0.95$ & 0.01 \\
\hline \multicolumn{12}{|c|}{ Soluble fibre } \\
\hline$n$ & \multicolumn{2}{|c|}{537} & \multicolumn{2}{|c|}{423} & \multicolumn{2}{|c|}{432} & \multicolumn{2}{|c|}{358} & \multicolumn{2}{|c|}{344} & \\
\hline & $1.00^{*}$ & Ref & $1.08^{*}$ & $0.75,1.54$ & $0.90^{*}$ & $0.62,1.31$ & $0.95^{\star}$ & $0.64,1.41$ & $0.68^{*}$ & $0.44,1.04$ & 0.06 \\
\hline & $1.00 \dagger$ & Ref & $0.88 \dagger$ & $0.69,1.13$ & $0.86 \dagger$ & $0.67,1.11$ & $0.95 \dagger$ & $0.73,1.24$ & $0.68 \dagger$ & $0.52,0.90$ & 0.02 \\
\hline & $1.00 \ddagger$ & Ref & $0.86 \ddagger$ & $0.67,1 \cdot 10$ & $0.85 \ddagger$ & $0.66,1 \cdot 10$ & $0.94 \ddagger$ & $0.72,1 \cdot 23$ & $0.69 \ddagger$ & $0.52,0.91$ & 0.03 \\
\hline \multicolumn{12}{|c|}{ Insoluble fibre } \\
\hline$n$ & \multicolumn{2}{|c|}{483} & \multicolumn{2}{|c|}{441} & \multicolumn{2}{|c|}{405} & & 98 & & 67 & \\
\hline & $1.00^{*}$ & Ref & $0.78^{\star}$ & $0.55,1.12$ & $0.76^{*}$ & $0.53,1.10$ & $0.69^{*}$ & $0.47,1.01$ & $0.49^{*}$ & $0.32,0.74$ & 0.001 \\
\hline & $1.00 \dagger$ & Ref & $0.91 \dagger$ & $0.71,1 \cdot 16$ & $0.87 \dagger$ & $0.67,1.12$ & $0.77 \dagger$ & $0.59,1.00$ & $0.66 \dagger$ & $0.51,0.86$ & 0.001 \\
\hline & $1.00 \ddagger$ & Ref & $0.91 \ddagger$ & $0.71,1.17$ & $0.87 \ddagger$ & $0.67,1.13$ & $0.78 \ddagger$ & $0.60,1.01$ & $0.67 \ddagger$ & $0.51,0.88$ & 0.003 \\
\hline Cere & ibre & & & & & & & & & & \\
\hline$n$ & & 19 & & 19 & & 18 & & 19 & & 19 & \\
\hline & $1.00^{*}$ & Ref & $0.91^{*}$ & $0.62,1.33$ & $0.89^{\star}$ & $0.61,1.31$ & $0.73^{*}$ & $0.49,1.09$ & $0.93^{*}$ & $0.64,1.36$ & $0.03 \ddagger$ \\
\hline & $1.00 \dagger$ & Ref & $0.86 \dagger$ & $0.67,1.11$ & $0.87 \dagger$ & $0.68,1.13$ & $0.65 \dagger$ & $0.50,0.84$ & $0.78 \dagger$ & $0.61,1.02$ & 0.04 \\
\hline & $1.00 \ddagger$ & Ref & $0.83 \ddagger$ & $0.64,1.07$ & $0.82 \ddagger$ & $0.63,1.06$ & $0.62 \ddagger$ & $0.47,0.81$ & $0.76 \neq$ & $0.58,0.99$ & 0.03 \\
\hline Vege & le fibre & & & & & & & & & & \\
\hline$n$ & & 19 & & 19 & & 18 & & 19 & & 19 & \\
\hline & $1.00^{*}$ & Ref & $0.81^{*}$ & $0.54,1.19$ & $0.79^{\star}$ & $0.53,1.16$ & $0.89^{*}$ & $0.60,1.30$ & $0.74^{*}$ & $0.50,1.10$ & 0.08 \\
\hline & $1.00 \dagger$ & Ref & $0.87 \dagger$ & $0.67,1.13$ & $0.78 \dagger$ & $0.60,1.02$ & $0.83 \dagger$ & $0.64,1.09$ & $0.77 \dagger$ & $0.59,1.00$ & 0.07 \\
\hline & $1.00 \ddagger$ & Ref & $0.88 \ddagger$ & $0.68,1.14$ & $0.80 \ddagger$ & $0.61,1.04$ & $0.85 \ddagger$ & $0.65,1.11$ & $0.79 \ddagger$ & $0.60,1.03$ & 0.12 \\
\hline Fruit & & & & & & & & & & & \\
\hline$n$ & & 19 & & 19 & & 18 & & 19 & & 19 & \\
\hline & $1.00^{*}$ & Ref & $0.88^{\star}$ & $0.60,1.28$ & $0.76^{\star}$ & $0.51,1.13$ & $0.72^{*}$ & $0.49,1.08$ & $0.69^{*}$ & $0.47,1.04$ & 0.08 \\
\hline & $1.00 \dagger$ & Ref & $0.71 \dagger$ & $0.55,0.92$ & $0.77 \dagger$ & $0.59,1.00$ & $0.71 \dagger$ & $0.54,0.92$ & $0.71 \dagger$ & $0.54,0.93$ & 0.12 \\
\hline & $1.00 \ddagger$ & Ref & $0.69 \ddagger$ & $0.53,0.90$ & $0.76 \ddagger$ & $0.58,0.99$ & $0.70 \ddagger$ & $0.53,0.91$ & $0.72 \ddagger$ & $0.55,0.95$ & 0.16 \\
\hline Seec & & & & & & & & & & & \\
\hline$n$ & & 19 & & 19 & & 18 & & 19 & & 19 & \\
\hline & $1.00^{*}$ & Ref & $0.91^{*}$ & $0.63,1.31$ & $1.03^{*}$ & $0.72,1.48$ & $0.56^{*}$ & $0.38,0.84$ & $0.57^{*}$ & $0.38,0.85$ & 0.03 \\
\hline & $1.00 \dagger$ & Ref & $1.03 \dagger$ & $0.80,1.33$ & $0.88 \dagger$ & $0.68,1.14$ & $0.81 \dagger$ & $0.63,1.05$ & $0.78 \dagger$ & $0.60,1.01$ & 0.05 \\
\hline & 1.00‡ & Ref & $0.92 \ddagger$ & $0 \cdot 70,1 \cdot 21$ & $0.76 \ddagger$ & $0.57,1.02$ & $0.71 \neq$ & $0.53,0.95$ & $0.73 \ddagger$ & $0.55,0.95$ & 0.05 \\
\hline Legu & fibre & & & & & & & & & & \\
\hline$n$ & & 19 & & 19 & & 18 & & 19 & & 19 & \\
\hline & $1.00^{*}$ & Ref & $0.75^{\star}$ & $0.51,1.10$ & $0.97^{\star}$ & $0.68,1.40$ & $0.70^{*}$ & $0.48,1.03$ & $0.65^{*}$ & $0.44,0.97$ & 0.01 \\
\hline & $1.00 \dagger$ & Ref & $0.88 \dagger$ & $0.68,1.13$ & $0.97 \dagger$ & $0.75,1.26$ & $0.83 \dagger$ & $0.64,1.08$ & $0.70 \dagger$ & $0.54,0.91$ & 0.01 \\
\hline & $1.00 \ddagger$ & Ref & $0.77 \ddagger$ & $0.58,1.02$ & $0.82 \ddagger$ & $0.61,1 \cdot 10$ & $0.74 \ddagger$ & $0.56,0.98$ & $0.63 \ddagger$ & $0.47,0.83$ & 0.003 \\
\hline
\end{tabular}

Ref, reference.

* Adjusted for age.

† Further adjusted for smoking and pack-years (among ever smokers).

‡ Further adjusted for educational level, leisure-time physical activity, total daily energy.

with asthma symptoms in both women and men (Tables 2 and 3).

\section{Sources of dietary fibre and asthma symptom score}

After adjustment for potential confounders, we observed a significant inverse association between cereal fibre, fruit fibre and seed fibre with asthma symptoms, both among women (Table 2) and men (Table 3), with OR for the highest quintile compared with the lowest quintile ranging from 0.66 to 0.85 . In women, after adjustment for potential confounders, vegetable fibre was also associated with lower asthma symptoms (OR for the highest quintile compared with the lowest quintile was 0.82 (95\% CI $0.76,0.89)$ ). Among men, the association was borderline significant for legume fibre (OR for the highest quintile compared with the lowest quintile was 0.89 (95\% CI
$0.77,1.03)$, with a significant trend ( $P$ for trend $=0.05)$. Overall, further adjustment for the mPNNS-GS did not substantially modify the association among both women and men (online Supplementary Tables S3 and S4).

\section{Types of dietary fibre and asthma control}

We observed a significant inverse association between higher dietary fibre intake and uncontrolled asthma, both among women and men (Fig. 2). After adjustments for potential confounders, OR for participants in the highest quintile was 0.72 (95\% CI $0.55,0.95)$ in women (Table 4) and 0.45 (95\% CI $0 \cdot 26,0 \cdot 79)$ in men (Table 5) compared with participants in the lowest quintile of total dietary fibre intake. Further adjustment for the mPNNS-GS did not modify the results (online Supplementary Table S5 and S6). 
Table 5. Associations between quintiles of dietary fibre intake and the asthma control test score among men from the NutriNet-Santé study ( $n$ 557) (Odds ratios and $95 \%$ confidence intervals)

\begin{tabular}{|c|c|c|c|c|c|c|c|c|c|c|c|}
\hline & \multicolumn{2}{|c|}{ Quintile 1} & \multicolumn{2}{|c|}{ Quintile 2} & \multicolumn{2}{|c|}{ Quintile 3} & \multicolumn{2}{|c|}{ Quintile 4} & \multicolumn{2}{|c|}{ Quintile 5} & \multirow[b]{2}{*}{$P$ for trend } \\
\hline & OR & $95 \% \mathrm{Cl}$ & OR & $95 \% \mathrm{Cl}$ & OR & $95 \% \mathrm{Cl}$ & OR & $95 \% \mathrm{Cl}$ & OR & $95 \% \mathrm{Cl}$ & \\
\hline \multicolumn{12}{|c|}{ Total fibre } \\
\hline$n$ & \multicolumn{2}{|c|}{137} & \multicolumn{2}{|c|}{101} & \multicolumn{2}{|c|}{111} & \multicolumn{2}{|c|}{108} & \multicolumn{2}{|c|}{100} & \\
\hline & $1.00^{*}$ & Ref & $0.69^{*}$ & $0.32,1.48$ & $0.49^{*}$ & $0.22,1.09$ & $0.47^{*}$ & $0.21,1.06$ & $0.31^{*}$ & $0.12,0.79$ & 0.001 \\
\hline & $1.00 \dagger$ & Ref & $0.81 \dagger$ & $0.49,1.33$ & $0.51 \dagger$ & $0.31,0.86$ & $0.62 \dagger$ & $0.37,1.04$ & $0.44 \dagger$ & $0.25,0.75$ & 0.002 \\
\hline & $1.00 \ddagger$ & Ref & $0.78 \ddagger$ & $0.47,1.30$ & $0.52 \ddagger$ & $0.31,0.88$ & $0.62 \ddagger$ & $0.36,1.06$ & $0.45 \ddagger$ & $0.26,0.79$ & 0.01 \\
\hline \multicolumn{12}{|c|}{ Soluble fibre } \\
\hline$n$ & \multicolumn{2}{|c|}{133} & \multicolumn{2}{|c|}{131} & \multicolumn{2}{|c|}{116} & \multicolumn{2}{|c|}{112} & \multicolumn{2}{|c|}{99} & \\
\hline & $1.00^{\star}$ & Ref & $0.65^{\star}$ & $0.32,1.35$ & $0.39^{\star}$ & $0.16,0.94$ & $0.33^{*}$ & $0.13,0.80$ & $0.40^{\star}$ & $0.17,0.95$ & 0.001 \\
\hline & $1.00 \dagger$ & Ref & $0.67 \dagger$ & $0.42,1.08$ & $0.56 \dagger$ & $0.33,0.95$ & $0.57 \dagger$ & $0.34,0.95$ & $0.42 \dagger$ & $0.24,0.73$ & 0.002 \\
\hline & $1.00 \ddagger$ & Ref & $0.65 \ddagger$ & $0.40,1.05$ & $0.54 \ddagger$ & $0.32,0.94$ & $0.57 \ddagger$ & $0.34,0.98$ & $0.44 \ddagger$ & $0.25,0.79$ & 0.01 \\
\hline \multicolumn{12}{|c|}{ Insoluble fibre } \\
\hline$n$ & \multicolumn{2}{|c|}{128} & \multicolumn{2}{|c|}{102} & \multicolumn{2}{|c|}{116} & & 2 & & 9 & \\
\hline & $1 \cdot 00^{*}$ & Ref & $0.22^{*}$ & $0.08,0.61$ & $0.58^{\star}$ & $0.28,1.20$ & $0.45^{\star}$ & $0.21,0.98$ & $0 \cdot 27^{\star}$ & $0.11,0.69$ & 0.01 \\
\hline & $1.00 \dagger$ & Ref & $0.60 \dagger$ & $0.36,1.00$ & $0.58 \dagger$ & $0.35,0.96$ & $0.50 \dagger$ & $0.30,0.84$ & $0.51 \dagger$ & $0.30,0.87$ & 0.02 \\
\hline & $1.00 \ddagger$ & Ref & $0.57 \ddagger$ & $0.34,0.96$ & $0.58 \ddagger$ & $0.35,0.97$ & $0.48 \ddagger$ & $0.28,0.81$ & $0.52 \ddagger$ & $0.30,0.90$ & 0.03 \\
\hline Cere & & & & & & & & & & & \\
\hline$n$ & & 111 & & 12 & & 11 & & 2 & & 11 & \\
\hline & $1.00^{*}$ & Ref & $1.43^{\star}$ & $0.65,3.18$ & $0.75^{\star}$ & $0.31,1.82$ & $0.83^{*}$ & $0.35,1.98$ & $0.92^{*}$ & $0.39,2.18$ & 0.31 \\
\hline & $1.00 \dagger$ & Ref & $0.94 \dagger$ & $0.56,1.57$ & $0.73 \dagger$ & $0.43,1.22$ & $0.94 \dagger$ & $0.56,1.56$ & $0.74 \dagger$ & $0.44,1.25$ & 0.33 \\
\hline & $1.00 \ddagger$ & Ref & $0.91 \ddagger$ & $0.54,1.53$ & $0.70 \ddagger$ & $0.41,1 \cdot 19$ & $0.91 \ddagger$ & $0.54,1.54$ & $0.75 \ddagger$ & $0.44,1.28$ & 0.38 \\
\hline Veg & le fibre & & & & & & & & & & \\
\hline$n$ & & 111 & & 12 & & 11 & & 2 & & 11 & \\
\hline & $1.00^{*}$ & Ref & $0.96^{*}$ & $0.43,2.14$ & $0.76^{*}$ & $0.33,1.75$ & $0.88^{*}$ & $0.39,1.96$ & $0.44^{*}$ & $0.17,1 \cdot 12$ & 0.01 \\
\hline & $1.00 \dagger$ & Ref & $1.01 \dagger$ & $0.61,1.68$ & $0.69 \dagger$ & $0.41,1.16$ & $0.61 \dagger$ & $0.36,1.03$ & $0.59 \dagger$ & $0.35,1.01$ & 0.01 \\
\hline & $1.00 \ddagger$ & Ref & $1.00 \ddagger$ & $0.60,1.66$ & $0.68 \ddagger$ & $0.40,1.15$ & $0.61 \ddagger$ & $0.36,1.03$ & $0.61 \ddagger$ & $0.36,1.04$ & 0.01 \\
\hline Fruit & & & & & & & & & & & \\
\hline$n$ & & 111 & & 12 & & 11 & & 2 & & 11 & \\
\hline & $1.00^{*}$ & Ref & $0.63^{*}$ & $0.29,1.38$ & $0.70^{*}$ & $0.32,1.52$ & $0.40^{*}$ & $0.17,0.95$ & $0.24^{*}$ & $0.10,0.64$ & 0.002 \\
\hline & $1.00 \dagger$ & Ref & $0.73 \dagger$ & $0.44,1.22$ & $0.76 \dagger$ & $0.46,1.28$ & $0.58 \dagger$ & $0.34,0.98$ & $0.36 \dagger$ & $0.20,0.64$ & 0.001 \\
\hline & $1.00 \ddagger$ & Ref & $0.69 \ddagger$ & $0.41,1 \cdot 16$ & $0.75 \ddagger$ & $0.44,1.28$ & $0.55 \ddagger$ & $0.32,0.95$ & $0.37 \ddagger$ & $0.21,0.67$ & 0.002 \\
\hline Seec & & & & & & & & & & & \\
\hline$n$ & & 111 & & 12 & & 11 & & 2 & & 11 & \\
\hline & $1.00^{\star}$ & Ref & $0.43^{\star}$ & $0.18,1.06$ & $0.80^{*}$ & $0.37,1.74$ & $0.88^{*}$ & $0.41,1.89$ & $0.45^{\star}$ & $0.19,1.09$ & 0.16 \\
\hline & $1.00 \dagger$ & Ref & $0.88 \dagger$ & $0.53,1.48$ & $0.88 \dagger$ & $0.52,1.48$ & $1.06 \dagger$ & $0.64,1.77$ & $0.67 \dagger$ & $0.40,1.15$ & 0.18 \\
\hline & $1.00 \ddagger$ & Ref & $0.76 \ddagger$ & $0.44,1.32$ & $0.71 \ddagger$ & $0.39,1.27$ & $0.85 \ddagger$ & $0.47,1.54$ & $0.61 \ddagger$ & $0.35,1.07$ & 0.18 \\
\hline Legu & fibre & & & & & & & & & & \\
\hline$n$ & & 111 & & 12 & & 11 & & & & 11 & \\
\hline & $1.00^{\star}$ & Ref & $1.09^{\star}$ & $0.49,2.43$ & $0.98^{*}$ & $0 \cdot 43,2 \cdot 21$ & $0.62^{*}$ & $0.25,1.53$ & $0.79^{\star}$ & $0.34,1.85$ & 0.22 \\
\hline & $1.00 \dagger$ & Ref & $1.08 \dagger$ & $0.64,1.80$ & $1.00 \dagger$ & $0.59,1.68$ & $0.78 \dagger$ & $0.46,1.32$ & $0.85 \dagger$ & $0.51,1.43$ & 0.19 \\
\hline & $1.00 \ddagger$ & Ref & $0.90 \ddagger$ & $0.52,1.57$ & $0.77 \ddagger$ & $0.42,1.39$ & $0.67 \ddagger$ & $0.38,1 \cdot 17$ & $0.77 \ddagger$ & $0.45,1.32$ & 0.20 \\
\hline
\end{tabular}

Ref, reference.

* Adjusted for age.

† Further adjusted for smoking and pack-years (among ever smokers).

‡ Further adjusted for educational level, leisure-time physical activity, total daily energy.

Regarding the fibre types, higher intake of both soluble and insoluble fibres was also significantly inversely associated with uncontrolled asthma in women (Table 4) and men (Table 5).

\section{Sources of dietary fibre and asthma control}

In women, after adjustment for confounders, we observed that greater intake of cereal, seed and legume fibre was significantly negatively associated with uncontrolled asthma (Table 4). In men, we observed significantly inverse association for vegetable and fruit fibre intake (Table 5). Further adjustment for the overall quality of the diet did not substantially change the results except for the legume fibre intake in women for which the association was no longer statistically significant (online Supplementary Tables S5 and S6).

\section{Discussion}

In this large study of French adults, we observed that higher dietary fibre intake (total, regardless of the type, namely, soluble and insoluble), was associated with less asthma symptoms in both women and men. Association was consistent in several sub-populations and after adjustment for several potential confounders. When specific sources of fibre were taken into consideration, intake of fibre from cereals, fruit and seeds was most consistently associated with less asthma symptoms. We also observed inverse association between uncontrolled asthma and greater intake of dietary fibre, mainly for total dietary fibre, soluble and insoluble fibres and fibre intake from cereals, fruit, seeds and legumes.

To our knowledge, this is the first study to investigate the association of both asthma symptoms and asthma control with 
total dietary fibre and types (soluble and insoluble) of fibre. Consistent with our findings, Berthon et al. ${ }^{(19)}$ showed that asthmatics with severe disease have an altered pattern of dietary intake with reduced total fibre intake, which was related to worsened lung function and airway inflammation, as compared with healthy controls. Likewise, the recent randomised controlled trial study conducted by Halnes et al. ${ }^{(20)}$ investigates the effect of a single dose of soluble fibre on airway inflammation biomarkers in stable asthma and reported a beneficial role of soluble fibre to reduce airway inflammation. Dietary fibre can be subcategorised by its solubility into water-insoluble and water-soluble fibres ${ }^{(44)}$; and although differences between these two types are mostly chemical and physical, and to a lesser extent physiological ${ }^{(45)}$, it is relevant to evaluate their separate effect on asthma. Our results extended previous findings regarding the role of soluble fibre and also suggested the role of insoluble fibre for both asthma symptoms and asthma control.

Overall, our findings regarding the role of specific sources of dietary fibre are in line with previous published studies showing that a healthy diet rich in fruits, vegetables or whole-grain cereals, and thus potentially rich in dietary fibre, may have beneficial role against asthma symptoms and asthma control ${ }^{(46-48)}$. However, those studies have not focused on fibre per se, and therefore, its effect might have been combined or diluted with other constituents of the diet with anti- or proinflammatory/antioxidant properties. Regarding associations between sources of fibre with various health outcomes such as CVD or COPD, significant results were mostly reported for cereal fibre ${ }^{(15,16,49)}$. In our study, besides cereal fibre, we also reported consistent significant associations between asthma symptoms and asthma control with various sources of dietary fibre including fruits and vegetables.

Asthma is a chronic inflammatory disease and several potential mechanisms underlying the dietary fibre-asthma association have been proposed, including inflammation and more recently, an imbalance in the gut microbiome. Regarding inflammation, several epidemiological studies have reported that a greater intake of total fibre was associated with lower level of C-reactive protein $(\mathrm{CRP})^{(50)}$ and various proinflammatory cytokines such as lower plasma levels of TNF- $\alpha$ receptor 2 and IL- $6{ }^{(51)}$. Consistently, a recent randomised controlled trial conducted by Honsek et al. reported a decrease in CRP level after 1 year intervention with insoluble fibre supplementation or promoting healthy diet (including among others, an increase in fibre intake $)^{(52)}$. In addition, in the recent pilot study by Halnes et $a l .^{(20)}$, when comparing participants with stable asthma to the control group, a higher consumption of soluble fibre was associated with lower levels of sputum neutrophils, macrophages and lymphocytes. However, since we also found significant association with insoluble fibre, our results may suggest that other mechanisms are likely to be involved. Likewise, a meta-analysis by Aune et al. reported a significant beneficial effects of diets rich in whole grain and high in insoluble fibre and also several health outcomes including respiratory inflammatory diseases ${ }^{(53)}$. Regarding microbiota, a growing number of investigators hypothesise that an imbalance in the gut microbiome caused by changes in the diet over the past decades may cause dysfunction of the immune system, leading to the development of asthma and allergy ${ }^{(18)}$.
Microbiota metabolise dietary soluble fibres through fermentation and decomposition and provide SCFA ${ }^{(54)}$. In children, the specific gut microbiota accompanied by a reduction of SCFA was associated with a higher risk of incident asthma ${ }^{(55)}$, and it has been shown that fibre intake altered the composition of both the gut and, to a lesser degree the lung, microbiota $^{(56)}$. In this line, high dietary fibre consumption might be relevant for primary and secondary prevention.

The present study has few limitations that should be considered. First, caution is needed when extrapolating our results since participants from the NutriNet-Santé cohort are all volunteers involved in a long-term cohort focused on nutrition and health and were overall more health conscious and interested in nutritional issue, with higher educational level and socioprofessional status ${ }^{(57)}$. Besides, proportion of men is low, which might have resulted in a relative lack of statistical power, especially when assessing the association between fibre intake and asthma control among men. Second, although dietary data were prospectively collected, the respiratory data used in our study were collected in a cross-sectional manner, limiting the conclusions that could be drawn with regard to causality. Nevertheless, the association remained significant in participants without ever asthma. Moreover, data were collected from selfreported questionnaires, which are more prone to biases and should also be interpreted with caution. However, self-reporting is widely used in epidemiological studies and, in the context of the NutriNet-Santé cohort, validation studies showed the accuracy of self-reported data ${ }^{(25,26,58)}$ along with reduced social desirability biases ${ }^{(59)}$. Third, given the observational design of our study, we cannot rule out the possibility of residual confounding. Fourth, potential overlap between asthma and COPD might also contribute to the association. However, it is unlikely since stratification by smoking status yielded consistent results among never smokers. Finally, high dietary fibre intake is one characteristic of a healthy diet, which was consistently reported to be associated with asthma, and, thus, other dietary components of these diets might also have contributed to the association. However, we reported similar associations when results were further adjusted for the overall nutritional quality of the diet.

Strengths of the present study include its sample size that gave us the possibility to perform stratified analyses, the availability of information about potential confounders and the fact that we could have estimated individual associations between asthma with total fibre, soluble and insoluble fibres or different sources of fibre. In addition, we used validated tools to assess asthma symptoms and asthma control. Finally, to better reflect usual intake, we used repeated 24-h dietary records over a long period to assess dietary data.

In conclusion, we observed that higher dietary fibre intake was associated with fewer asthma symptoms and greater asthma control. Overall, our results suggested that dietary fibre might play an important role in the prevention of asthma and its lack of control and thus reinforce the growing evidence of benefit of dietary fibre for lung health. Furthermore, these findings lent further support for public health nutrition policies and practices that encourage healthy eating behaviours with an increased consumption of dietary fibre, mostly for insoluble fibres or fibres from cereals. 


\section{Acknowledgements}

The authors thank Younes Esseddik, Thi Hong Van Duong, Paul Flanzy, Régis Gatibelza, Jagatjit Mohinder (computer scientists), Cédric Agaësse (dietitian), Julien Allègre, Nathalie Arnault, Laurent Bourhis and Fabien Szabo de Edelenyi, PhD (datamanagers/biostatisticians) and Fatoumata Diallo, MD (physician). We thank all the volunteers of the NutriNet-Santé cohort.

The NutriNet-Santé Study was supported by the following public institutions: Ministère de la Santé, Santé Publique France, Institut National de la Santé et de la Recherche Médicale, Institut National de la Recherche Agronomique, Conservatoire National des Arts et Métiers and Université Paris 13.

Author contributions were as follows. R. M. A., P. G. and R. V. designed and conducted the research; E. K. G., N. D. P., M. T., S. H., P. G. and R. V. provided essential reagents or provided essential materials; R. M. A., and R. V. analysed data or performed statistical analysis; R. M. A., P. G. and R. V. wrote the manuscript and had primary responsibility for the final content; R. M. A., S. H., E. K. G., N. D. P., M. T., P. G. and R. V. were involved in interpreting the results and editing the manuscript for important intellectual content. All authors read, edited and approved the final manuscript.

The authors have no conflicts of interest to declare.

\section{Supplementary material}

For supplementary materials referred to in this article, please visit https://doi.org/10.1017/S0007114519001843

\section{References}

1. Haut Conseil de la Santé Publique (2017) Statement Related to the Revision of the 2017-2021 French Nutrition and Health Programme's Dietary Guidelines for Adults. Paris: HCSP.

2. US Department of Health and Human Services and US Department of Agriculture (2015) Dietary Guidelines for Americans 2015-2020. https://health.gov/DietaryGuidelines/ 2015/guidelines/ (accessed October 2018).

3. Wang S-S, Lay S, Yu H-N, et al. (2016) Dietary guidelines for Chinese residents (2016): comments and comparisons. J Zhejiang Univ Sci B 17, 649-656.

4. Slavin JL \& Lloyd B (2012) Health benefits of fruits and vegetables. Adv Nutr 3, 506-516.

5. Esmaillzadeh A, Kimiagar M, Mehrabi Y, et al. (2006) Fruit and vegetable intakes, C-reactive protein, and the metabolic syndrome. Am J Clin Nutr 84, 1489-1497.

6. Hosseini B, Berthon BS, Wark P, et al. (2017) Effects of fruit and vegetable consumption on risk of asthma, wheezing and immune responses: a systematic review and meta-analysis. Nutrients 9, 341.

7. Mamluk L, O'Doherty MG, Orfanos P, et al. (2017) Fruit and vegetable intake and risk of incident of type 2 diabetes: results from the consortium on health and ageing network of cohorts in Europe and the United States (CHANCES). Eur J Clin Nutr $\mathbf{7 1}$, 83-91.

8. Huang T, Xu M, Lee A, et al. (2015) Consumption of whole grains and cereal fiber and total and cause-specific mortality: prospective analysis of 367,442 individuals. BMC Med 13, 59.
9. Chen G-C, Tong X, Xu J-Y, et al. (2016) Whole-grain intake and total, cardiovascular, and cancer mortality: a systematic review and meta-analysis of prospective studies. Am J Clin Nutr 104, 164-172.

10. Veronese N, Solmi M, Caruso MG, et al. (2018) Dietary fiber and health outcomes: an umbrella review of systematic reviews and meta-analyses. Am J Clin Nutr 107, 436-444.

11. Weickert MO \& Pfeiffer AFH (2018) Impact of dietary fiber consumption on insulin resistance and the prevention of type 2 diabetes. J Nutr 148, 7-12.

12. World Cancer Research Fund/American Institute for Cancer Research (2018) Diet, nutrition, physical activity and cancer: A global perspective. Continuous Update Project Expert Report 2018. The Third Expert Report. dietandcancerreport. org (accessed January 2019).

13. Jacobs DR, Andersen LF \& Blomhoff R (2007) Whole-grain consumption is associated with a reduced risk of noncardiovascular, noncancer death attributed to inflammatory diseases in the Iowa Women's Health Study. Am JClin Nutr 85, 1606-1614.

14. Hanson C, Lyden E, Rennard S, et al. (2016) The relationship between dietary fiber intake and lung function in the National health and nutrition examination surveys. Ann Am Thorac Soc 13, 643-650.

15. Kaluza J, Harris H, Wallin A, et al. (2018) Dietary fiber intake and risk of chronic obstructive pulmonary disease: a prospective cohort study of men. Epidemiol Camb Mass 29, 254-260.

16. Varraso R, Willett WC \& Camargo CA (2010) Prospective study of dietary fiber and risk of chronic obstructive pulmonary disease among US women and men. Am J Epidemiol 171, 776-784.

17. Butler LM, Koh W-P, Lee H-P, et al. (2004) Dietary fiber and reduced cough with phlegm: a cohort study in Singapore. Am J Respir Crit Care Med 170, 279-287.

18. McKenzie C, Tan J, Macia L et al. (2017) The nutrition-gut microbiome-physiology axis and allergic diseases. Immunol Rev 278, 277-295.

19. Berthon BS, Macdonald-Wicks LK, Gibson PG et al. (2013) Investigation of the association between dietary intake, disease severity and airway inflammation in asthma: diet and asthma. Respirology 18, 447-454.

20. Halnes I, Baines K, Berthon B, et al. (2017) Soluble fibre meal challenge reduces airway inflammation and expression of GPR43 and GPR41 in asthma. Nutrients $9,57$.

21. Boudier A, Curjuric I, Basagaña X, et al. (2013) Ten-year followup of cluster-based asthma phenotypes in adults. A pooled analysis of three cohorts. Am J Respir Crit Care Med 188, 550-560.

22. Sunyer J, Pekkanen J, Garcia-Esteban R, et al. (2007) Asthma score: predictive ability and risk factors. Allergy 62, 142-8.

23. Pekkanen J (2005) Operational definitions of asthma in studies on its aetiology. Eur Respir J 26, 28-35.

24. Hercberg S, Castetbon K, Czernichow S, et al. (2010) The Nutrinet-Santé study: a web-based prospective study on the relationship between nutrition and health and determinants of dietary patterns and nutritional status. BMC Public Health 10, 242.

25. Lassale C, Castetbon K, Laporte F, et al. (2015) Validation of a web-based, self-administered, non-consecutive-day dietary record tool against urinary biomarkers. BrJ Nutr 113, 953-962.

26. Lassale C, Castetbon K, Laporte F, et al. (2016) Correlations between fruit, vegetables, fish, vitamins, and fatty acids estimated by web-based nonconsecutive dietary records and respective biomarkers of nutritional status. J Acad Nutr Diet 116, 427-438.e5.

27. Touvier M, Kesse-Guyot E, Méjean C, et al. (2011) Comparison between an interactive web-based self-administered $24 \mathrm{~h}$ dietary record and an interview by a dietitian for large-scale epidemiological studies. Br J Nutr 105, 1055-1064. 
28. Le Moullec N, Deheeger M, Preziosi P, et al. (1996) Validation of the photo manual used for the collection of dietary data in the SU.VI.MAX. Study. Cah Nut Diét 31, 158-164.

29. Black AE (2000) Critical evaluation of energy intake using the Goldberg cut-off for energy intake:basal metabolic rate. A practical guide to its calculation, use and limitations. Int J Obes Relat Metab Disord J Int Assoc Study Obes 24, 1119-1130.

30. Schofield WN (1985) Predicting basal metabolic rate, new standards and review of previous work. Hum Nutr Clin Nutr 39, Suppl. 1, 5-41.

31. Etude Nutrinet-Santé (2013) Table de Composition des Aliments de L'Étude Nutrinet-Santé (Nutrinet-Santé Study Food Composition Database). Paris: Economica.

32. Willett WC, Howe GR \& Kushi LH (1997) Adjustment for total energy intake in epidemiologic studies. Am J Clin Nutr 65 , 1220S-1228S; discussion 1229S-1231S.

33. Estaquio C, Kesse-Guyot E, Deschamps V, et al. (2009) Adherence to the French Programme National Nutrition Santé guideline score is associated with better nutrient intake and nutritional status. J Am Diet Assoc 109, 1031-1041.

34. Burney PG, Luczynska C, Chinn S, et al. (1994) The European community respiratory health survey. Eur Respir J 7, 954-960.

35. Andrianasolo RM, Kesse-Guyot E, Adjibade M, et al. (2018) Associations between dietary scores with asthma symptoms and asthma control in adults. Eur Respir J 52, 1702572

36. Schatz M, Sorkness CA, Li JT, et al. (2006) Asthma control test: Reliability, validity, and responsiveness in patients not previously followed by asthma specialists. J Allergy Clin Immunol 117, 549-556.

37. World Health Organization (2000) Obesity: preventing and managing the global epidemic. Report of a WHO consultation. World Health Organ Tech Rep Ser 894, 1-253.

38. Hagströmer M, Oja P \& Sjöström M (2006) The International physical activity questionnaire (IPAQ): a study of concurrent and construct validity. Public Health Nutr 9, 755-762.

39. Fernstrand AM, Bury D, Garssen J, et al. (2017) Dietary intake of fibers: differential effects in men and women on perceived general health and immune functioning. Food Nutr Res 61, 1297053.

40. Jacquemin B, Sunyer J, Forsberg B, et al. (2009) Association between modelled traffic-related air pollution and asthma score in the ECRHS. Eur Respir J 34, 834-842.

41. Anderson JW, Baird P, Davis RH, et al. (2009) Health benefits of dietary fiber. Nutr Rev 67, 188-205.

42. Bédard A, Dumas O, Kauffmann F, et al. (2012) Potential confounders in the asthma-diet association: how causal approach could help? Allergy 67, 1461-1462; author reply 1462-1463.

43. Yuan YC (2000) Multiple imputation for missing data: Concepts and new development. In Proceedings of the Twenty-Fifth Annual SAS Users Group International Conference, 9-12 April 2000, p. 267. Cary, NC: SAS Institute.

44. Dhingra D, Michael M, Rajput $\mathrm{H}$, et al. (2012) Dietary fibre in foods: a review. J Food Sci Technol 49, 255-266.
45. Dikeman CL \& Fahey GC (2006) Viscosity as related to dietary fiber: a review. Crit Rev Food Sci Nutr 46, 649-663.

46. Ma J, Strub P, Lv N, et al. (2016) Pilot randomised trial of a healthy eating behavioural intervention in uncontrolled asthma. Eur Respir J 47, 122-132.

47. Wood LG, Garg ML, Smart JM, et al. (2012) Manipulating antioxidant intake in asthma: a randomized controlled trial. $\mathrm{Am} \mathrm{J}$ Clin Nutr 96, 534-543.

48. Li Z, Kesse-Guyot E, Dumas O, et al. (2017) Longitudinal study of diet quality and change in asthma symptoms in adults, according to smoking status. Br J Nutr 117, 562-571.

49. Cho SS, Qi L, Fahey GC, et al. (2013) Consumption of cereal fiber, mixtures of whole grains and bran, and whole grains and risk reduction in type 2 diabetes, obesity, and cardiovascular disease. Am J Clin Nutr 98, 594-619.

50. Ma Y, Griffith JA, Chasan-Taber L, et al. (2006) Association between dietary fiber and serum C-reactive protein. $A m \mathrm{~J}$ Clin Nutr 83, 760-766.

51. Ma Y, Hébert JR, Li W, et al. (2008) Association between dietary fiber and markers of systemic inflammation in the women's health initiative observational study. Nutrition 24, 941-949.

52. Honsek C, Kabisch S, Kemper M, et al. (2018) Fibre supplementation for the prevention of type 2 diabetes and improvement of glucose metabolism: the randomised controlled Optimal Fibre Trial (OptiFiT). Diabetologia 61, 1295-1305.

53. Aune D, Keum N, Giovannucci E, et al. (2016) Whole grain consumption and risk of cardiovascular disease, cancer, and all cause and cause specific mortality: systematic review and dose-response meta-analysis of prospective studies. BMJ 353, i2716.

54. Ríos-Covián D, Ruas-Madiedo P, Margolles A, et al. (2016) Intestinal short chain fatty acids and their link with diet and human health. Front Microbiol 7, 185.

55. Arrieta M-C, Stiemsma LT, Dimitriu PA, et al. (2015) Early infancy microbial and metabolic alterations affect risk of childhood asthma. Sci Transl Med 7, 307ra152.

56. Trompette A, Gollwitzer ES, Yadava K, et al. (2014) Gut microbiota metabolism of dietary fiber influences allergic airway disease and hematopoiesis. Nat Med 20, 159-166.

57. Andreeva VA, Deschamps V, Salanave B, et al. (2016) Comparison of dietary intakes between a large online cohort study (Etude NutriNet-Santé) and a nationally representative cross-sectional study (Etude Nationale Nutrition Santé) in France: addressing the issue of generalizability in E-epidemiology. Am J Epidemiol 184, 660-669.

58. Lassale C, Péneau S, Touvier M, et al. (2013) Validity of webbased self-reported weight and height: results of the Nutrinet-Santé study. J Med Internet Res 15, e152.

59. Kesse-Guyot E, Assmann K, Andreeva V, et al. (2016) Lessons learned from methodological validation research in E-epidemiology. JMIR Public Health Surveill 2, e160. 